УДК 004.4'2

DOI: $10.15827 / 0236-235 X .125 .109-114$
Дата подачи статьи: 10.09 .18

2019. T. 32. № 1. C. 109-114

\title{
Компьютерное моделирование физических взаимодействий технических поверхностей на микроуровне
}

\author{
A.A. Рачишкин 1, к.т.н., доиент, RachishkinAndr@yandex.ru \\ A.Н. Болотов 1, д.m.н., nрофрессор, зав. кафредрой, alnikbltov@rambler.ru \\ O.B. Сутягин 1, д.т.н., доиент, sutyagine@rambler.ru \\ 1 Тверской государственный технический университет, г. Тверь, 170026, Россия
}

\begin{abstract}
В статье представлена архитектура программного средства для моделирования физических взаимодействий технических поверхностей на микроуровне, описываются общие принципы построения системы и проводится анализ нескольких смоделированных физических процессов. Рассматривается контактное взаимодействие шероховатых поверхностей, в том числе и имеющих функциональное покрытие. Рассчитываются фрикционные параметры при различных условиях эксплуатации и термическое сопротивление микрошероховатых стыков различных деталей машин.

Гибкий алгоритм программы и независимый сегментированный математический аппарат разработаны для оптимальной реализации расчетов моделируемых процессов численными методами. Входные данные основываются на микрогеометрических и физико-механических свойствах реальных поверхностей, что позволяет точно настраивать модель, учитывая большинство свойств, характеризующих технические поверхности. Создание программного средства для моделирования контактного взаимодействия технических поверхностей позволяет облегчить решение прикладных инженерных задач и уменьшает количество необходимых ресурсов для проведения научных исследований.

Структурно программа разделена на отдельные модули. Формулирование общих принципов разработки каждого модуля эффективно при горизонтальном расширении системы моделирования физических взаимодействий. Такой подход позволяет оптимизировать количество входных и выходных параметров, настраивать только необходимые процессы моделирования и привносит возможность добавлять и изменять существующие алгоритмы. Модульная структура оптимизирует процесс разработки и модернизации программного приложения. Возможность удалять и модифицировать отдельные элементы программы, не затрагивая общую структуру, позволяет эффективно решать широкий спектр инженерных задач.

В статье приведены примеры работы модулей контактного и фрикционного взаимодействий, а также моделирования термического сопротивления стыков. Общая алгоритмическая логика выполнения и наследуемые данные топографии поверхности позволяют максимально приближенно моделировать данные физические процессы.
\end{abstract}

Ключевые слова: компьютерное моделирование, дискретный контакт, функциональные покрытия, микронеровность, контактное взаимодействие, фрикиионное взаимодействие, термическое сопротивление.

На сегодняшний день для решения большинства сложных технических задач, в частности, при расчетах характеристик контактного взаимодействия шероховатых поверхностей, широко распространены и применяются аналитические методы [1-3]. Однако эти методы имеют ряд допущений, которых можно избежать, объединив их и современные информационные технологии. Разработанное программное средство для моделирования шероховатых поверхностей позволит исключить такие ситуации: стей;

- непрерывное распределение микронеровно-

- замена трехмерного пространства на двумерHoe;

- замена микронеровностей сферическими элементами с постоянным радиусом;

- отсутствие возможностей контроля и редактирования геометрических и физико-механических свойств как самих микронеровностей, так и моделируемых физических процессов.

Программное средство для моделирования физических взаимодействий технических поверхностей дает возможность облегчить решение конструкторских задач и уменьшить количество ресурсов для проведения комплексных научных исследований. Его главная задача - прогнозирование физических процессов и опти- мизация моделируемых систем. При создании такого программного средства необходимо учесть особенности поставленной задачи и определить общую структуру программы, разбив ее на отдельные модули с единой системой записи, чтения, обработки и хранения данных. Данный подход оптимальный, поскольку предусматривается дальнейшее расширение функциональных возможностей системы из-за широкого спектра решаемых практических задач. Базовыми модулями системы являются генерация поверхности [4] и контактное взаимодействие. Остальные элементы слабо зависят друг от друга и объединены только общей структурой хранения данных. При таком подходе можно удалять, изменять и редактировать отдельные сегменты системы, не затрагивая ничего лишнего. Также допускается наличие двух модулей с однотипной задачей, но использующих различные варианты аналитических решений. Это необходимо для тестирования влияния тех или иных параметров на конечный результат моделирования. Для реализации каждого модуля следует сформулировать общие принципы их построения.

Общие принципы построения модулей. При разработке каждого модуля ставится и адекватно решается ряд последовательных задач: 
- формулирование цели;

- разработка алгоритмов и математического аппарата для их реализации;

- тестирование компьютерной модели.

Единая структурная логика позволяет осуществлять горизонтальное расширение системы, модернизировать имеющиеся компоненты и обеспечивает об- щую логику работы программы. Алгоритм отображен на рисунке 1.

Система ввода входных параметров связана с функционалом помощи пользователю для обеспечения ввода адекватных параметров. Это настраиваемый инструмент, не допускающий как связанных с человеческим фактором ошибок, так и логически невозмож-

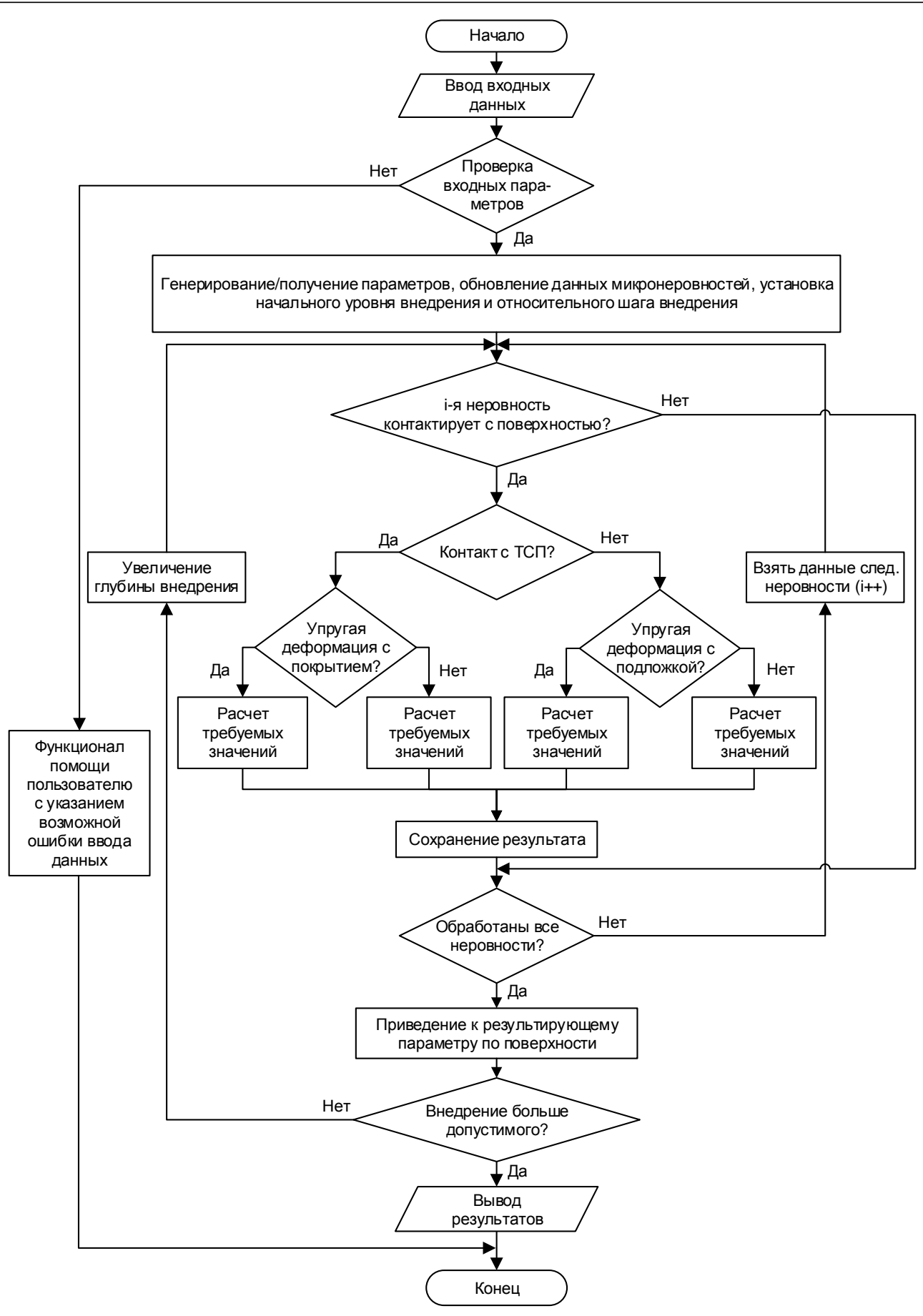

Рис. 1. Блок-схема общей логики построения модулей

Fig. 1. Block diagram of the general module building logic 
ных ситуаций, заданных разработчиком модуля. Такой подход облегчает работу оператора и упрощает процесс его взаимодействия с системой. Микрогеометрические и физико-механические параметры, получаемые в результате экспериментальных исследований, обрабатываются и передаются непосредственно в блоки обработки данных. По входным данным осуществляется генерирование параметров моделируемых технических поверхностей. Такая логика взаимодействия создана из-за вариативности и изменяемости моделируемых ситуаций. При изменении одного из входных параметров необязательно проводить перерасчет всей системы, достаточно корректировочного моделирования зависимых от данного параметра блоков. Временные и ресурсные затраты при этом существенно сокращаются.

Далее идет процесс последовательной проверки контактного взаимодействия каждой микрошероховатости с поверхностью. При отсутствии контакта неровность исключается из процесса моделирования на данном шаге внедрения. Если проверка положительная, осуществляется анализ контакта неровности. Этот подход разработан для данной модели и учитывает как максимальное количество индивидуальных параметров самой неровности, так и ситуации, в которой происходит взаимодействие. Анализируются наличие твердосмазочного покрытия и характер деформации. Математическая модель строится с учетом каждой из возможных алгоритмических ситуаций. После обработки всех неровностей производится приведение к результирующему параметру по всей поверхности на данном шаге внедрения, что позволяет максимально точно сопоставить моделируемые и реальные данные. Затем система рассчитывает следующий шаг внедрения, задаваемый пользователем. Если внедрение допустимо, итерация цикла продолжается, иначе выводятся общие результаты процесса моделирования в формате, заданном разработчиком.

Модуль контактного взаимодействия. Результаты синтеза трехмерной модели шероховатой поверхности используются при моделировании контактного взаимодействия с упругим полупространством, имеющим относительно мягкое упругопластическое покрытие [5]. Сгенерированная поверхность обладает приведенными параметрами шероховатости и соответствует случаю контакта двух шероховатых поверхностей $[6,7]$. Допускается, что сегменты эллипсоидов абсолютно жесткие. Благодаря архитектуре программы толщина и физико-механические свойства покрытия можно динамически изменять по случайному закону распределения либо задавать постоянными для моделирования однородной поверхности. Для расчета локального износа на случайных участках поверхности введен специальный коэффициент износа от 0 до $100 \%$. При $100 \%$ рассматриваются ситуации взаимодействия контактных поверхностей без покрытий.

При описании математической модели анализируются варианты контактного взаимодействия с упругим полупространством, имеющим упругопластическое покрытие, и с упругопластическим полупространством без покрытия. Возникающие деформации проходят проверку и рассчитываются либо как упругие, либо как упругопластические. Таким образом, расчет нагрузки для всех неровностей, находящихся в контакте, можно записать в виде

$$
N=\sum_{i=1}^{j} N_{1 i e}+\sum_{i=1}^{k} N_{1 i e p}+\sum_{i=1}^{l} N_{2 i e}+\sum_{i=1}^{q} N_{2 i e p},
$$

где $N$ - нормальная нагрузка, приложенная к шероховатой поверхности; $N_{1 i e}-$ нагрузки на сегменты, контактирующие с покрытием при его упругой деформации и упругой деформации основания; $N_{1 i e p}-$ нагрузки на сегменты, контактирующие с покрытием при его упругопластической деформации и упругой деформации основания; $N_{2 i e}$ - нагрузки на сегменты, контактирующие с основанием при его упругой деформации; $N_{2 i e p}$ - нагрузки на сегменты, контактирующие с основанием при его упругопластической деформации; $j$ количество сегментов, контактирующих с покрытием при его упругой деформации и упругой деформации основания; $k$ - количество сегментов, контактирующих с покрытием при его упругопластической деформации и упругой деформации основания; $l$ - количество сегментов, контактирующих с основанием при его упругой деформации; $q$ - количество сегментов, контактирующих с основанием при его упругопластической деформации. Данную задачу можно решить численным методом при пошаговом внедрении [8].

Необходимые для расчетов входные параметры модуль Юнга, коэффициент Пуассона, микротвердость - вводятся оператором и являются статистически обработанными механическими свойствами.

Тестирование модуля проводилось по экспериментальным данным, полученным в $[7,8]$. Результат показал хорошую сходимость при моделировании всех экспериментальных ситуаций. Отклонение минимально и не выходит за пределы погрешности. На рисунке 2 приведены результаты компьютерного моделирования контактного взаимодействия шероховатых поверхностей с твердосмазочным покрытием и соответствующие им экспериментальные данные [8]. Параметры покрытий: а) золото $\delta=16$ мкм; б) кадмий $\delta=30$ мкм; в) кадмий $\delta=3$ мкм; г) серебро $\delta=30$ мкм; д) ФБФ74-Д $\delta=20$ мкм; е) ФБФ74-Д $\delta=12$ мкм.

Необходимо отдельно рассмотреть рисунок 2в. На нем экспериментальные данные из работы [8] с покрытием из кадмия толщиной 3 мкм. Как видно из графика, соответствие моделирования значительно лучше при принятии ситуации, когда 90 \% микронеровностей контактируют с твердосмазочным покрытием, а $10 \%$ с основанием. Это можно объяснить большой вероятностью непосредственного контакта микронеровностей контртела с основанием.

Модуль фрикционного взаимодействия. Модуль является подключаемым и наследует предыдущие этапы моделирования. 

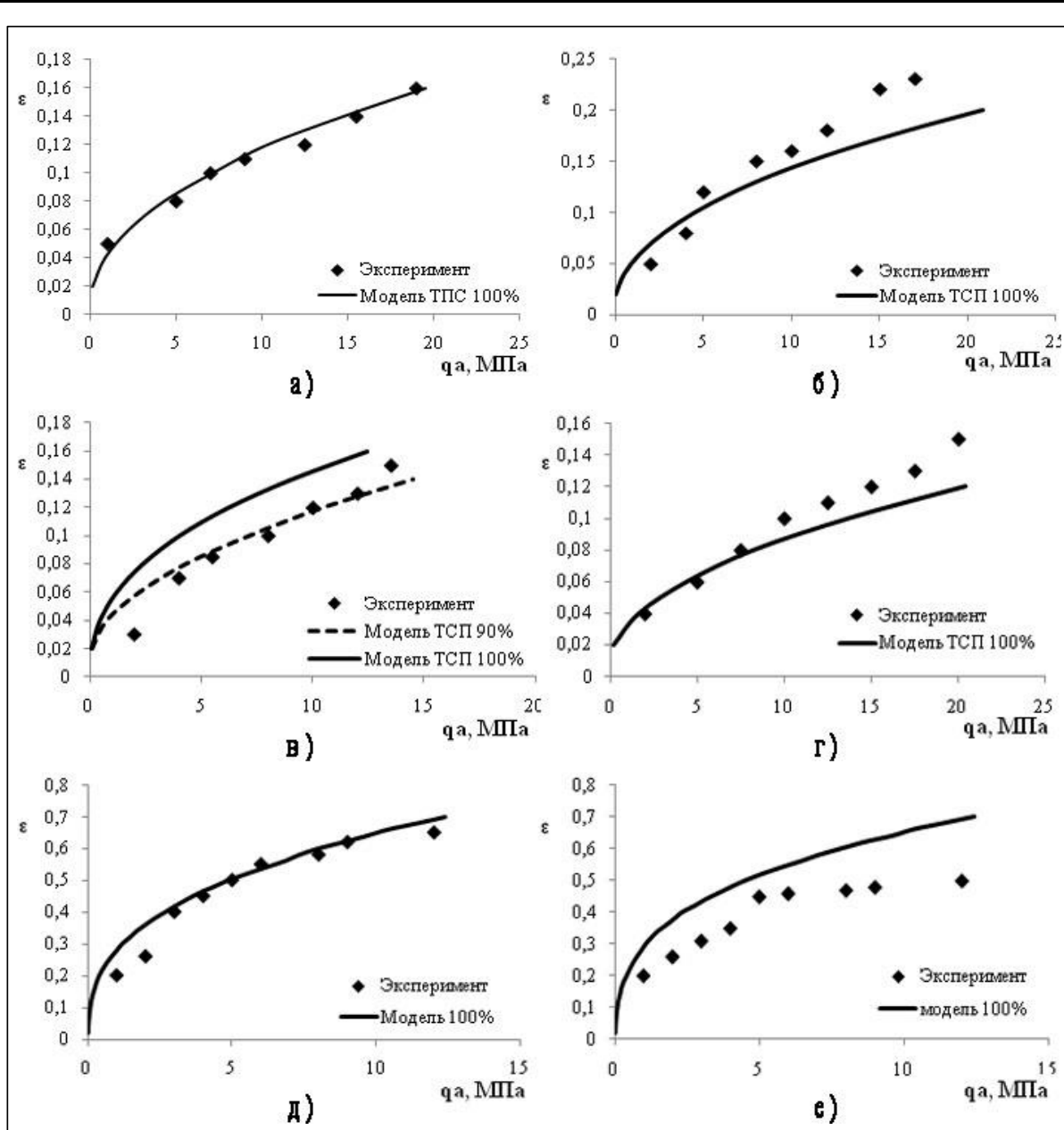

Рис. 2. Зависимости для относительного сближения стальных шероховатых образцов со стальныли образцами, имеющими функциональное или твердосмазочное покрытие

Fig. 2. Dependencies for the relative convergence of rough steel and steel samples that have a functional or solid lubricating coating

Результирующую силу трения для сегментов, находящихся в контакте, можно выразить в виде

$$
F=\sum_{i=1}^{j} F_{1 i e}+\sum_{i=1}^{t} F_{1 i e p}+\sum_{i=1}^{l} F_{2 i e}+\sum_{i=1}^{q} F_{2 i e p},
$$

где $F$ - суммарная сила трения; $F_{1 i e}-$ силы трения на сегментах, контактирующих с покрытием при его упругой деформации; $F_{1 i e p}-$ силы трения на сегментах, контактирующих с покрытием при его упругопластической деформации; $F_{2 i e}-$ силы трения на сегментах, контактирующих с основанием при его упругой деформации; $F_{2 i e p}-$ силы трения на сегментах, контактирующих с основанием при его упругопластической деформации; $j$ - количество сегментов, контактирующих с покрытием при его упругой деформации; $t$ - количество сегментов, контактирующих с покрытием при его упругопластической деформации; $l$ - количество сегментов, контактирующих с полупространством при его упругой деформации; $q$ - количество сегментов, контактирующих с полупространством при его упругопластической деформации.
Входные параметры принимаются из общего интерфейса программы, поэтому параметры микрогеометрии, физико-механические свойства исследуемых материалов и покрытий уже присутствуют на данном этапе моделирования. Предусмотрено изменение одного или нескольких параметров из вышестоящих модулей. При этом необходимо провести корректирующие перерасчеты характеристик поверхностей и покрытий. Блочная структура программы позволяет не проводить новый синтез шероховатой поверхности при изменении механических свойств материалов.

Входные параметры, используемые в данном модуле:

- тангенциальная прочность молекулярных связей на границе сегмент-покрытие (не используется при расчетах коэффициента трения трибосопряжения без покрытия, не вводится первый параметр);

- тангенциальная прочность молекулярных связей на границе сегмент-основание (не используется при моделировании трибосопряжения с покрытием, без учета параметра локального износа тангенциальной прочности молекулярных связей на границе сегмент-основание).

Тестирование модуля проводилось с использованием экспериментальных данных работы [9] и собственных экспериментальных данных о влиянии контактных давлений на статический коэффициент трения (рис. 3). При исследовании использовались образцы с твердосмазочным покрытием ВНИИНП-230 толщиной 0,03 мм (маркеры - ромбы), ВНИИНП-212 0,03 мм (маркеры - квадраты) и ВНИИНП-212 - 0,005 мм (маркеры - треугольники). Представленные экспериментальные данные удовлетворительно соответствуют результатам компьютерного моделирования (прозрачные маркеры соответствующих для марок фигур с твердосмазочным покрытием).

Модуль термического сопротивления. Модуль является подключаемым и наследует предыдущие этапы моделирования.

Термическое сопротивление дискретного контакта можно представить как совокупность параллельных термических сопротивлений единичных пятен контакта, сформировавшихся под действием внешней 
нагрузки. Следовательно, для теплонагруженного шероховатого контакта термическое сопротивление $R_{\text {шт. }}$ можно определить как

$$
\frac{1}{R_{\text {шт. }}}=\sum_{i=1}^{n_{r}} \frac{1}{R_{\text {пт } i}},
$$

где $n_{r}$ - число микронеровностей, вступивших в контакт с покрытием; $R_{\text {пті }}$ - термическое сопротивление единичного пятна контакта с покрытием.

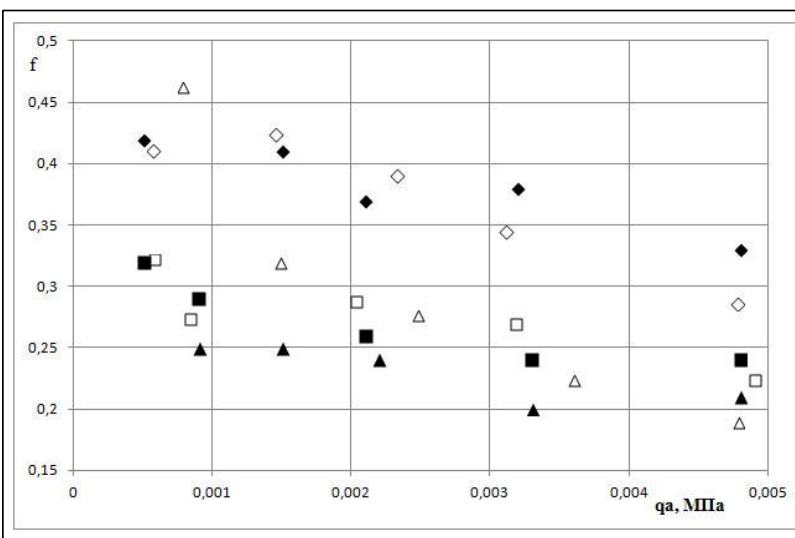

Рис. 3. Сопоставление экспериментальных значений коэффициента трения с результатами компьютерного моделирования

Fig. 3. Comparison of the friction coefficient experimental values with computer simulation results

Входными параметрами, задаваемыми оператором для расчета термического сопротивления стыков, являются значения теплопроводности контактирующих материалов. В случае наличия композитного покрытия берутся минимальное и максимальное значения его локальной теплопроводности и параметры бета-распределения для обеспечения неоднородности материала. Аналогично модулю фрикционного взаимодействия расчет контактируемых сегментов проводится в требуемый момент времени по формуле (3).

Тестирование проводилось по численным расчетам работы [10] и экспериментальным данным [11]. На рисунке 4 показаны зависимости термического сопротивления контакта от давления (эксперименты по исследованию образцов без покрытий). Контурными маркерами показаны результаты компьютерного моделирования, заполненными - экспериментальные данные.

Представленные результаты также показывают хорошую сходимость экспериментальных и моделируемых данных по зависимостям термического сопротивления от номинального давления для шероховатых стыков без покрытий.

Заключение. Разработана и реализована компьютерная программа, имеющая модульную архитектуру, позволяющая дополнять и модернизировать свое поведение в зависимости от требований оператора. Оптимизация и ориентация на объектную структуру дают
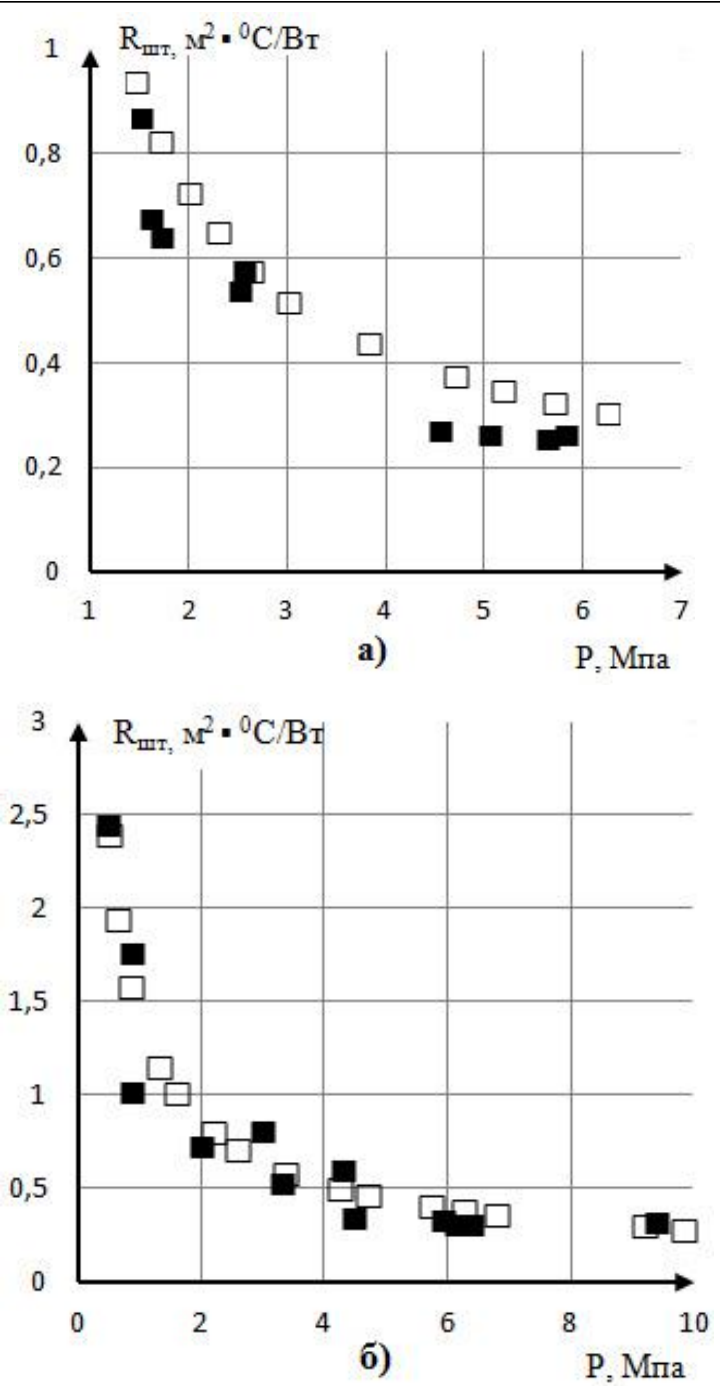

Рис. 4. Сопоставление экспериментальных данных с моделируемыми: нерж. сталь 18/8 / нерж. сталь 18/8:

а) чистовое шллифование; б) предварительное шлифование

Fig. 4. Comparison of the experimental data with the simulated data: stainless steel 18/8 / stainless steel 18/8: a) final grinding; б) pre-grinding

возможность адекватно описывать топографию поверхности без потребления большого количества ресурсов ЭВМ и проводить моделирование различных физико-механических процессов быстро и без аппаратных сбоев. Наличие инструмента, влияющего на вариативность входных параметров, помогает подобрать реальную шероховатую поверхность, оптимально подходящую по своим микрогеометрическим и физико-механическим свойствам для поставленной инженерной задачи.

\section{Лuтература}

1. Greenwood J.A., Williamson J.B.P. Contact of nominally flat surfaces. Proc. Roy. Soc., ser. A, 1966, vol. 295, no. 1442, pp. 300-319. 
2. Goryacheva I.G. Mechanics of discrete contact. Tribology Intern., 2006, vol. 39, no. 5, pp. 381-386.

3. Goryacheva I. Multiscale modelling in contact mechanics. IUTAM Simposiums, Springer, 2008, pp. 123-134.

4. Сутягин О.В., Болотов А.Н., Рачишкин А.А. Компьютерное моделирование микротопографии шероховатых поверхностей // Трение и износ. 2015. Т. 36. № 5. С. 536-545.

5. Крагельский И.В., Добычин М.Н., Комбалов В.С. Основы расчетов на трение и износ. М.: Машиностоение, 1977. 526 с.

6. Сутягин О.В., Болотов А.Н., Рачишкин А.А. Компьютерное моделирование контактного взаимодействия шероховатых поверхностей // Трение и износ. 2015. Т. 36. № 5. С. 536-545.
7. Демкин Н.Б. Контактирование шероховатых поверхностей. М.: Наука, 1970. 223 с.

8. Алексеев Н.М. Металлические покрытия опор скольжения М.: Наука, 1973. 75 с.

9. Гриб В.В., Лазарев Г.Е. Лабораторные испытания материалов на трение и износ. М.: Наука, 1968. 141 с.

10. Dryden J.R. The effect of a surface coating on the constriction resistance of a spot on an in nite half plane. J. Heat Transfer, 1983, vol. 105 , no. 2 , pp. 408-410.

11. Thomas T.R., Probert S.D. Correlations for thermal contact conductance in vacuum. Trans. Am. Soc. Mech. Engrs. 1972, vol. 94, pp. 176-180.

\section{Computer simulation of physical interactions of technical surfaces at the micro-level}

A.A. Rachishkin ${ }^{1}$, Ph.D. (Engineering), Accociate Professor, RachishkinAndr@yandex.ru

A.N. Bolotov ${ }^{1}$, Dr.Sc. (Engineering), Professor, Head of Chair, alnikbltov@ rambler.ru

O.V.Sutyagin ${ }^{1}$, Dr.Sc. (Engineering), Accociate Professor, sutyagine@ rambler.ru

${ }^{1}$ Tver State Technical University, Nikitin Quay 22, Tver, 170026, Russian Federation

Abstract. The paper presents the software design for computer simulation of physical interactions of engineering surfaces at the micro level. It describes general principles of the system and analyses several simulated physical processes. It also considers contact interaction of rough surfaces including those with functional coatings. It calculates frictional parameters under various operating conditions and the thermal resistance of microindented joints of various machine parts.

A flexible algorithm of the program and an independent segmented mathematical logic are developed for the optimal calculation of simulated processes using numerical methods. Input data is based on micro-geometric and physical-mechanical properties of real surfaces. This allows adjusting the model considering most technical surface properties. Developing software for simulation of contact interaction of technical surfaces makes it easier to solve engineering tasks and reduces the amount of resources needed for research.

The software is divided into separate modules. The definition of general principles for each module development is beneficial for the horizontal scaling of a system for modeling physical interactions. This approach allows optimizing the number of input and output parameters, adding and modifying existing algorithms and configuring only necessary modeling processes. A modular structure optimizes the software development process. Due to the ability to remove and modify individual program modules without affecting the overall structure, it is possible to solve a wide range of engineering tasks effectively.

The paper gives some examples of module operation for contact and friction interactions, as well as modeling thermal resistance of joints. The general algorithmic logic and the inherited surface topography data allow modeling these physical processes as real as possible.

Keywords: computer modelling, discrete contact, functional coatings, microroughness, contact interaction, frictional interaction, thermal resistance.

\section{References}

1. Greenwood J.A., Williamson J.B.P. Contact of nominally flat surfaces. Proc. Roy. Soc., ser. A. 1966, vol. 295, no. 1442, pp. 300-319.

2. Goryacheva I.G. Mechanics of discrete contact. Tribology Intern. 2006, vol. 39, no. 5, pp. 381-386.

3. Goryacheva I.G. Multiscale modelling in contact mechanics. IUTAM Simp. Springer Publ., 2008, pp. $123-134$.

4. Sutyagin O.V., Bolotov A.N., Rachishkin A.A. Computer simulation of rough-surface microtopography. J. of Friction and Wear. 2015, vol. 36, no. 5, pp. 409-416 (in Russ.).

5. Kragelsky V.I., Dobychin M.N., Kombalov V.S. Basics of Friction and Wear Calculations. Moscow, Mashinostroenie Publ., 1977,516 p.

6. Sutyagin O.V., Bolotov A.N., Rachishkin A.A. Computer simulation of contact interaction of rough surfaces. J. of Friction and Wear. 2016, vol. 37, no. 3, pp. 198-203 (in Russ.).

7. Demkin N.B. Coating Rough Surfaces. Moscow, Nauka Publ., 1970, 223 p.

8. Alekseev N.M. Metal Coatings for Sliding Bearings. Moscow, Nauka Publ., 1973, 75 p.

9. Grib V.V., Lazarev G.E. Materials Laboratory Testing for Friction and Wear. Moscow, Nauka Publ., 1968,141 p.

10. Dryden J.R. The effect of a surface coating on the constriction resistance of a spot on an in nite half plane. J. of Heat Transfer. 1983, vol. 105, no. 2, pp. 408-410.

11. Thomas T.R., Probert S.D. Correlations for thermal contact conductance in vacuum. Trans. Am. Soc. Mech. Engrs. 1972 , vol. 94, pp. 176-180. 